\title{
Inspection Detectability Improvement for Metal Defects Detected by Pulsed Infrared Thermography
}

\author{
Zhengwei YANG ${ }^{1,2^{*}}$, Guangjie KOU ${ }^{1}$, Yin $\mathrm{LI}^{1}$, Gan TIAN ${ }^{1}$, Wei ZHANG ${ }^{1}$, and Jietang ZHU ${ }^{1}$ \\ ${ }^{1}$ Rocket Force University of Engineering, Xi'an 710025, China \\ ${ }^{2}$ School of Mechanical Engineering, Xi'an Jiaotong University, Xi'an 710049, China \\ *Corresponding author: Zhengwei YANGＥ-mail: yangzhengwei1136@163.com
}

\begin{abstract}
Aiming at the drawbacks of low contrast and high noise in the thermal images, a novel method based on the combination of the thermal image sequence reconstruction and the first-order differential processing is proposed in this work, which is comprised of the following procedures. Firstly, the specimen with four fabricated defects with different sizes is detected by using pulsed infrared thermography. Then, a piecewise fitting based method is proposed to reconstruct the thermal image sequence to compress the data and remove the temporal noise of each pixel in the thermal image. Finally, the first-order differential processing based method is proposed to enhance the contrast. An experimental investigation into the specimen containing de-bond defects between the steel and the heat insulation layer is carried out to validate the effectiveness of the proposed method via the above procedures. The obtained results show that the proposed method can remove the noise, enhance the contrast, and even compress the data reaching at $99.1 \%$, thus improving the detectability of pulsed infrared thermography on metal defects.
\end{abstract}

Keywords: Pulsed infrared thermography; metal defects; detectability improvement; piecewise fitting; differential processing

Citation: Zhengwei YANG, Guangjie KOU, Yin LI, Gan TIAN, Wei ZHANG, and Jietang ZHU, "Inspection Detectability Improvement for Metal Defects Detected by Pulsed Infrared Thermography," Photonic Sensors, 2019, 9(2): 142-150.

\section{Introduction}

Owning to the advantages of high speed, high efficiency, non-contact, and visible inspection results, the pulsed infrared thermography nondestructive technique has been successfully applied to evaluate defects for materials, especially for metals [1-3]. However, metals have the characteristics of excellent heat conductivity, leading to the sharply changing rate of temperature. Additionally, there are some problems of non-uniform heat and surface reflection. All of these cause the thermal images with the drawbacks of low contrast and high noise, which embarrasses the evaluation of defects. Thus, it is vital to enhance the contrast and remove the noise for facilitating the evaluation of defect.

As for the contrast enhancement, X. P. Maldague et al. [4] proposed several types of contrasts based methods, e.g., absolute contrast, normalized contrast, and standard contrast, to enhance the image quality, but these methods are limited to the definition of the sound area. To solve this problem, M. Pilla et al. [5] developed a differentiated absolute contrast (DAC) method to successfully perform on the semi-infinite materials, but with low calculation accuracy and

Received: 22 January 2018 / Revised: 4 July 2018

(C) The Author(s) 2019. This article is published with open access at Springerlink.com

DOI: $10.1007 / \mathrm{s} 13320-019-0489-1$

Article type: Regular 
with the exception of considering the thickness of the object. Thus, involving the thickness of the object, C. I. Castanedo et al. [6] modified the DAC method based on the thermal quadrupoles theory, which was proven as a great method able to improve the contrast of thermal image and calculation accuracy. Additionally, Guo et al. [7] proposed such methods as deleting fitted background, polynomial fitting, and integrated technique to enhance the contrast that was caused by non-uniform heating. As for the noise removal, R. Usamentiaga et al. [8] utilized the short Fourier transform to process the thermal image and improved the signal-to-noise ratio (SNR). Q. J. Tang et al. [9] proposed a method by combining the Markov chain with principal component analysis to perform an excellent capability to improve SNR. Moreover, several methods including independence component analysis [10], wavelet decomposition [11], mathematical morphology [12], etc. are applied to remove noise in thermal images. However, most of previous references available are devoted to the single frame image, and the relationships between the thermal image sequences are hardly involved.

On this account, a novel method based on the combination of thermal image sequence reconstruction and differential processing is proposed to enhance the contrast and remove the noise. To this goal, this work begins with the detection on the defects in the metal using pulsed infrared thermography, followed by theoretic analysis on the root and character of the noise in the thermal image sequence. On this basis, the piecewise fitting based method is proposed to reconstruct the thermal image sequence. Then, the first-order differential is used for processing the thermal image. The proposed method is validated by another specimen containing de-bond defects, showing excellent performance.

\section{Experiment for specimen 1\# using pulsed infrared thermography}

\subsection{Experiment preparation}

The specimen $1 \#$ is a steel plate (Fig. 1) with dimensions of $280 \mathrm{~mm} \times 100 \mathrm{~mm} \times 6 \mathrm{~mm}$ and four fabricating flat-bottom hole defects. The depth of the four defects is $4 \mathrm{~mm}$, and the diameters are $20 \mathrm{~mm}$, $15 \mathrm{~mm}, 10 \mathrm{~mm}$, and $5 \mathrm{~mm}$, respectively.

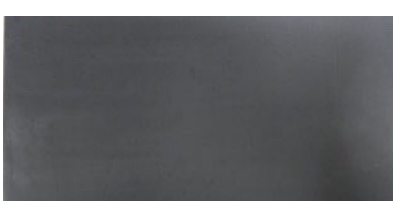

(a)

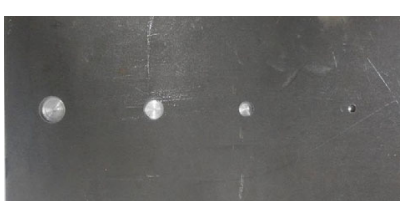

(b)
Fig. 1 Specimen $1 \#$ with four flat-bottom hole defects: (a) front surface and (b) back surface.

Experiment is done by the pulsed infrared thermography system (Fig. 2) exploited by us, which mainly includes two high-power flash lights, an infrared camera, and a computer. A flash light is used to release the pulse thermal wave and heat the specimen to produce temperature gradient, an infrared camera with high performance is used to measure and record the surface temperature distribution, and a computer is used to acquire and process temperature images of the specimen surface.

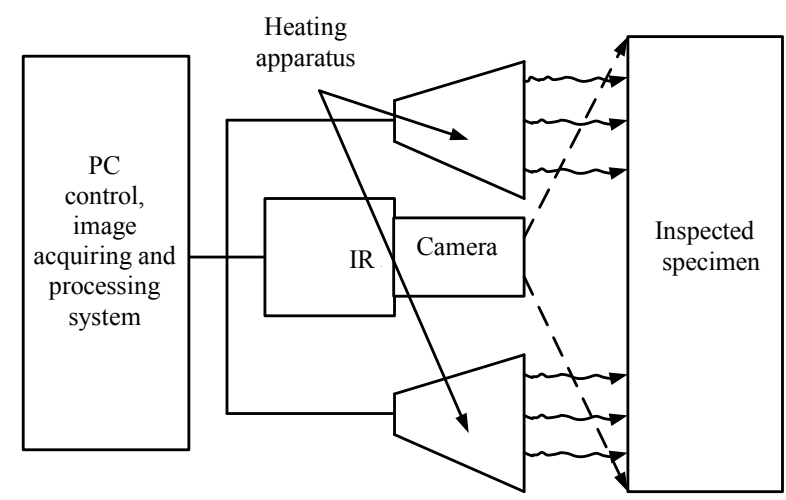

Fig. 2 Pulsed infrared thermography system.

In the experiment, the energy of the thermal wave is $9600 \mathrm{~J}$, and the duration time is $2 \mathrm{~ms}$. In addition, the acquiring rate of the infrared camera is set to $60 \mathrm{~Hz}$. 


\subsection{Experimental results and analysis}

The raw thermal image sequence acquired in the experiment is shown in Fig. 3. Four defects represented by hot spots appear first at the time of $0.12 \mathrm{~s}$. Subsequently, the hot spots gradually present clearly. As the heat transforms transversely, the temperature distributes uniformly, and hot spots gradually blur. The bigger the defect size is, the longer the hot spot lasts on the thermal image sequence, indicating that the more obvious the hot spot presents, the easier the defect is to be detected. However, the hot spot of the defect in the metal material does not hold strong contrast owing to the little temperature difference between the defect area and sound area. Therefore, for enhancing the defect contrast, one way is to increase the temperature difference between the defect and sound area, and another way is to remove the noise in the sound area to increase the SNR.
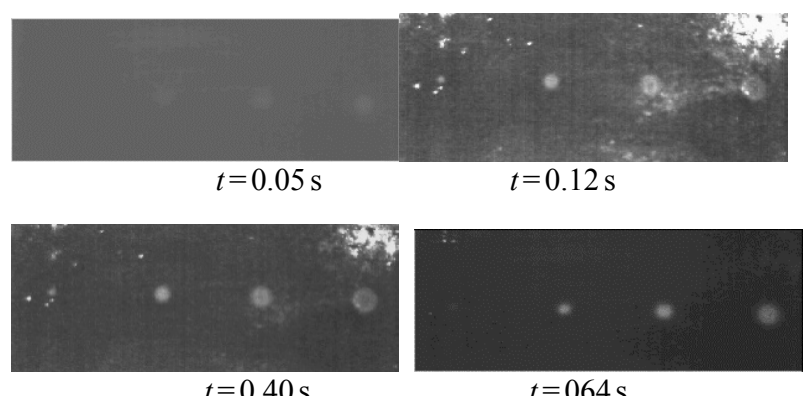

$t=0.40 \mathrm{~s}$

$t=064 \mathrm{~s}$

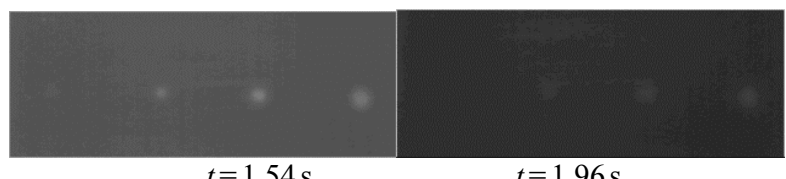

Fig. 3 Raw thermal images sequence of the specimen 1\#.

\subsection{Noise analysis}

From the above experiment, it can be seen that the noise plays an important role in the evaluation of defects, since it may blur the edge of the hot spots and make the evaluation of defect parameters more difficult. In order to remove the noise caused by the non-uniform of the metal surface, the non-uniform of the heating loading distribution, the detection environment, and other random noise in complicated manners, a kind of black paint is brushed on the surface of the specimen. Such operation can efficiently depress the reflection of the heat and improve SNR of the defect. However, the defect with a smaller size can still hardly be found in the raw thermal image sequence.

In fact, noises in the thermal image sequence include multiplicative noise and additive noise. Multiplicative noise is attributed to the non-uniform surface emissivity of the specimen caused by the contaminated or scraggy specimen surface condition, which may be partially eliminated by coating black paint with uniform emissivity on the specimen surface in the experiment. Additive noise is always caused by non-uniform heating, camera noise, the noise due to surrounding heat sources, and random noise. In the experiment, an aluminium cabinet is used to eliminate the noise caused by the reflections from other heating sources, and non-uniform heating also can be eliminated by the later signal processing such as the fast Fourier transform (FFT). The system noise and random noise exist everywhere and always cannot be avoided inevitably. For a certain frame, the noise namely spatial noise could be eliminated by using standard image enhancement methods. But for a certain pixel, the noise namely temporal noise changes with time, which needs to be eliminated by filtering methods.

\section{Thermal image sequence reconstruction}

In order to remove the noise and improve defect detectability, data processing methods have to be adopted in the first instance. In this work, a novel thermal image sequence reconstruction method is proposed based on piecewise fitting, which contains the following two steps:

Step 1: fit the temperature sequence of every pixel in the thermal image;

Step 2: reconstruct thermal image sequence based on the fitting coefficient obtained by Step 1 .

Figure 4 illustrates the temperature changes of the specimen surface, which can be divided into 
three segments:

(1) Segment with a fast temperature increase after the pulse heating. In this segment, the surface temperature of the specimen increases quickly and reaches a maximum value of $44.6{ }^{\circ} \mathrm{C}$. Consistent with the interval of the pulse heating, this segment consumes only several micro seconds.

(2) Segment with a fast temperature decrease. Due to the heat transformation into the specimen from the surface, heat convection, and radiation, the surface temperature of the specimen decreases quickly.

(3) Segment with a smooth temperature decrease. Consuming a relatively longer interval, this segment involves the occurrence of the temperature difference between the defect and other areas.



Fig. 4 Surface temperature varies after pulse excitation.

In order to fit the temperature changes precisely, the fitting operations are divided into three parts based on the above analysis, and the fitting results are shown in Fig. 5.

In the segment with a fast temperature increase, the whole increase process consumes $2 \mathrm{~ms}$, and the collected sampling data are little, because the sampling frequency is $60 \mathrm{~Hz}$. However, an increase segment phase is not the key point of the detection, a linear function is used to fit the data, and the result is given as follows:

$$
T(t)=21.836+11392 t \quad 0 \leq t \leq 0.002 .
$$

In the segment with a fast temperature decrease, one can fit the data with exponential function. In order to improve the fitting accuracy, triple exponential function is used to fit the data, and the corresponding result is given as follows:

$$
\begin{aligned}
T(t)= & 22.62284+5.71796 \mathrm{e}^{-\frac{t}{0.01529}}+39.04273 \mathrm{e}^{-\frac{t}{0.00502}}+ \\
& 1.96405 \mathrm{e}^{-\frac{t}{0.07737}}, \quad 0.002<t<0.2 .
\end{aligned}
$$
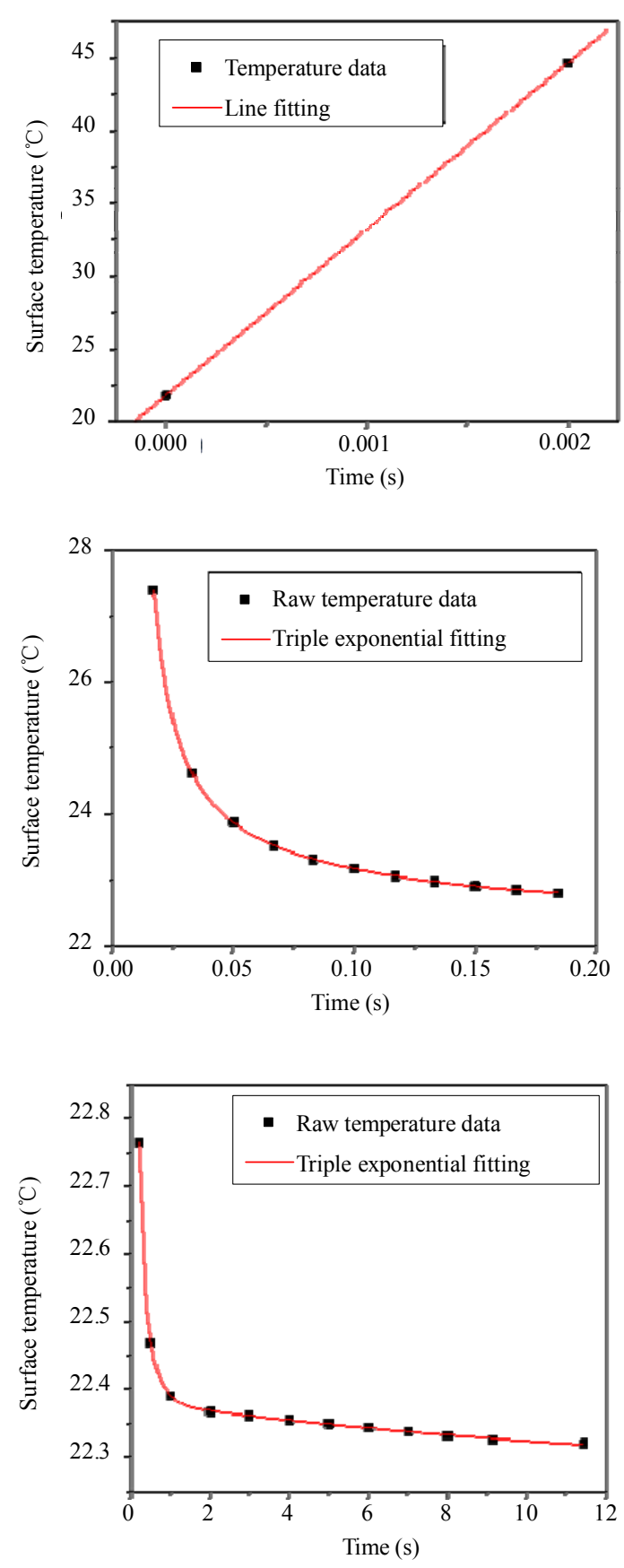

Fig. 5 Subsection fitting results of the surface temperature data. 
Similarly, a triple exponential function is used to fit the temperature changes in the segment with a smooth temperature decrease, and the result is given as follows:

$$
\begin{aligned}
& T(t)=22.26562+0.41536 \mathrm{e}^{-\frac{t}{0.3041}}+1.16752 \mathrm{e}^{-\frac{t}{0.10305}}+ \\
& 0.11809 \mathrm{e}^{-\frac{t}{14.27144}}, \quad 0.2<t<12 .
\end{aligned}
$$

Figure 6 illustrates the fitting results of the temperature curve in the whole interval. Compared with the raw data, it can be seen that the fitting curve fits the data with excellent accuracy.



Fig. 6 Temperature curves of the primitive and the reconstructed.

Similarly, all pixel points of the thermal image sequence can be fitted, and the thermal image sequence can be reconstructed by the fitting coefficients. As shown in Fig. 7, it can be seen that the fitting curve holds less noise than the raw data. Furthermore, the fitting method can save much storage space. For example, if the sampling frequency of thermal image is $60 \mathrm{~Hz}$, and the collected interval lasts $30 \mathrm{~s}$, a storage of $138.24 \mathrm{MB}$ is needed for the raw thermal image when the image holds a resolution of $320 \times 240$ pixel. However, only the storage of $1.2288 \mathrm{MB}$ is needed if the raw data are fitted and restored 16 coefficients of the fitting curve at every pixel, which saves the storage by $99.1 \%$ and facilitates the subsequent image processing.

Figure 8 illustrates the reconstructed image obtained by the fitting curve of the temperature at every pixel point. It can be seen that the reconstructed image, holding primary characters of the raw image, has less noise than the raw image, and the needed storage space has also been reduced.



Fig.7 Fitting of temperature sequence containing pixel noise.

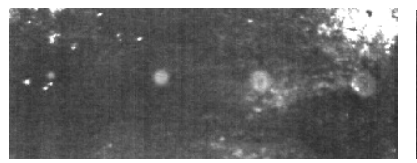

(a)



(b)
Fig. 8 Thermal image reconstructed by the piecewise fitting method: (a) raw image and (b) reconstructed image.

From the above investigation, it can be concluded that the piecewise fitting reconstruction method can remove the noise to some degree as well as compress the temperature data and decrease the storage space, which paves the way for facilitating the subsequent thermal image processing.

\section{Contrast enhancement based on the first-order differential processing}

Even if the reconstructed sequences have a lower noise, they contain strong background interference. If the signal is very weak, it is difficult for the operator to identify. Based on the study and analysis of the original image, we present a method based on the first-order differential processing to enhance the contrast and improve the detection capability.

Infrared thermal images acquired from the experiment represent the surface temperature distribution of the inspected material after pulse heating. In the one-dimensional condition, the temperature of the specimen surface is determined as 


$$
T_{\text {sound }}(0, t)=\frac{q}{\sqrt{\pi \rho c \lambda t}}
$$

where $T_{\text {sound }}$ is the surface temperature on the sound area. When the specimen contains some defects, the temperature on the defect surface is determined by

$$
T_{\text {corr }}(0, t)=\frac{q}{\sqrt{\pi \rho c \lambda t}}\left(1+\sum_{n=1}^{\infty} \mathrm{e}^{-\frac{(2 n h)^{2}}{4 \alpha t}}\right)
$$

where $T_{\text {corr }}$ is the surface temperature above the defect area, $q$ denotes the heating energy, $c$ denotes the specific heat, $\lambda$ denotes the thermal conductivity of the detected material, $\alpha$ denotes the thermal diffusivity of the detected material, $h$ denotes the depth of the defect, and $t$ denotes time.

From (4) and (5), it can be seen that the defect will affect the temperature distribution of the specimen surface, and the hot spot in the thermal image contains the information of defect such as location, size, and depth. In order to improve the contrast between the defect area and the sound area on the thermal image, the differentials of (4) and (5) are calculated as follows:

$$
\begin{gathered}
\frac{d T_{\text {sound }}(t)}{d t}=-\frac{1}{2} \frac{q}{\sqrt{\pi \rho c \lambda}} t^{-\frac{3}{2}} \\
\frac{d T_{\text {corr }}(t)}{d t}=-\frac{q}{\sqrt{\pi \rho c \lambda}} \cdot \\
{\left[-\frac{1}{2} t^{-\frac{3}{2}}\left(1+\sum_{n=1}^{\infty} \mathrm{e}^{-\frac{(2 n h)^{2}}{4 \alpha t}}\right)+\frac{n^{2} h^{2}}{\alpha} t^{-\frac{5}{2}} \sum_{n=1}^{\infty} \mathrm{e}^{-\frac{(2 n h)^{2}}{4 \alpha t}}\right] .}
\end{gathered}
$$

Based on the fitting function of the surface temperature, we can obtain the differential of the temperature curve at every pixel. The temperature curve and corresponding differential of both defect and the other area are shown in Fig. 9.

From Fig. 9(a), it can be seen that the surface temperature of the defect area is bigger than that of the sound area before processing using the first-order differential. However, the decrease speed of the surface temperature is different at the defect area and the sound area [in Fig. 9(b)]. At the beginning, the gray value of the sound area is bigger than that of the defect area. However, after the point of the intersection of the temperature curves, the gray value of the defect area is bigger than that of the sound area. As the time passes by, the gray values of both the defect and sound area converge together.

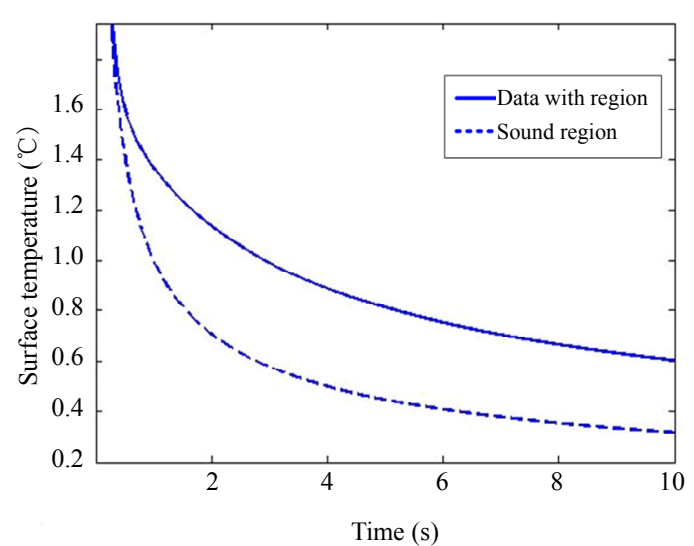

(a)

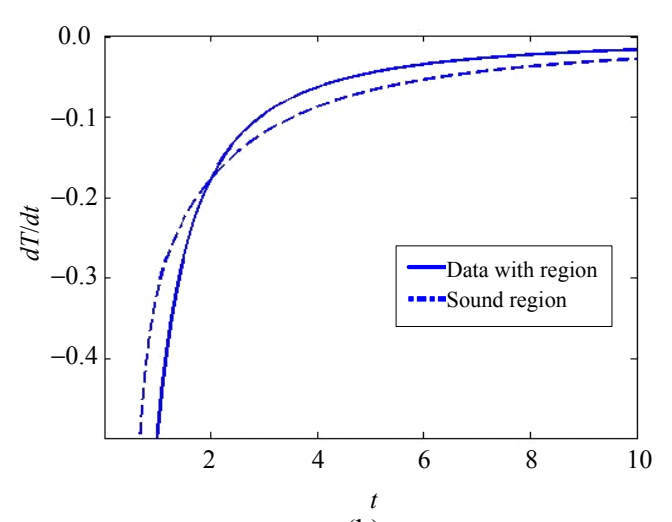

(b)

Fig. 9 Corresponding regional temperature of the defection area and its differential curve: (a) surface temperature curves and (b) first-order differential curve of the temperature on the surface.

The thermal image sequence obtained by the first-order differential method is shown in Fig. 10. It can be seen that the gray values of the defect and the sound area inter-change at about $0.18 \mathrm{~s}$. The moment, corresponding to the point of the intersection of the differential of the temperature curves, is the best suitable time for the defect detection, i.e., the temperature difference between the defect and the other area reaches the maximum value at the intersectin point of the temperature curve. Therefore, the images subtraction performed before and after 
the reversal of the gray value of the image can be used to improve the contrast of the image. Figure 11 illustrates the image obtained by the sutraction of the image before and after the gray reserval. It can be seen that the noise around the defect area has been removed, and the contrast between the defect and the sound area has been enhanced, because the hot spot of the defect with the smallest size is obvious in the image, which indicates the first-order differential processing method can enhance the contrast of thermal image.

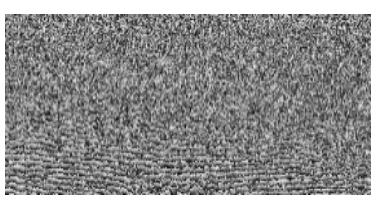

$t=0.04 \mathrm{~s}$

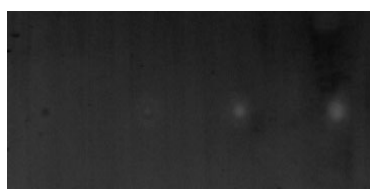

$t=0.18 \mathrm{~s}$

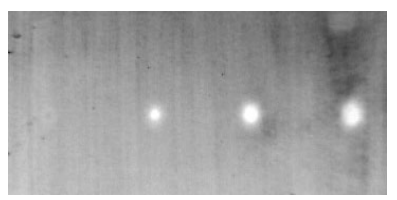

$t=0.12 \mathrm{~s}$

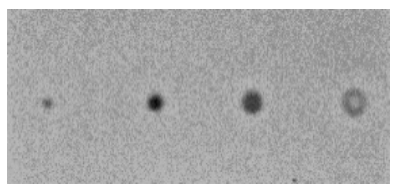

$t=0.44 \mathrm{~s}$
Fig. 10 Thermal image sequence after using the first-order differential processing.

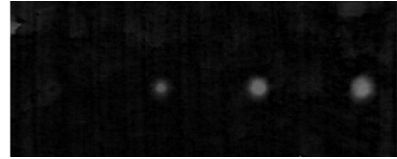

(a)

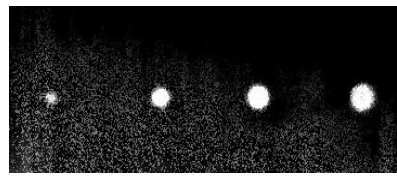

(c)

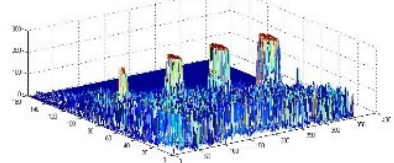

(e)



(b)



(d)

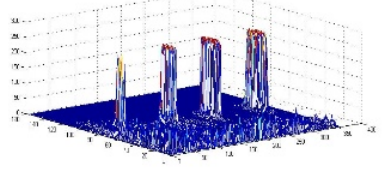

(f)
Fig. 11 Results of the subtraction process using the first-order differential processing: (a) subtract backgroud before the gray reversal, (b) subtract background after the gray reversal, (c) images in (a) subtract image (b), (d) denoise, (e) results of the three dimensional image of image (c), (f) results of the three dimensional image of (d).

\section{Experimental validation performed by the specimen $2 \#$}

In order to validate the efficiency of the proposed method, an experiment is performed by using the specimen $2 \#$ containing the de-bond defects between the steel and the heat insulation layer (Fig. 12), and the corresponding results are processed by the proposed method:

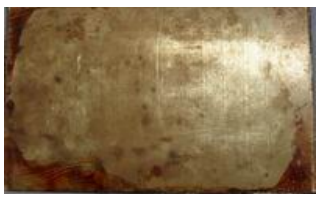

(a)

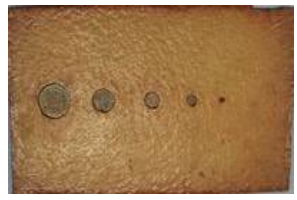

(b)
Fig. 12 Photo of the specimen 2\#: (a) front surface and (b) back surface.

(1) Thermal images sequence reconstruction of the specimen $2 \#$

The raw thermal image sequence is reconstructed based on the piecewise fitting method, and the corresponding results are shown Fig. 13. It can be seen that the surface of the specimen holds a uniform temperature distribution at the beginning, although the whole area is influenced by heavy noise. However, with time passing by, it is clearly seen that two hot spots, i.e. two defects, are appeared first at $0.38 \mathrm{~s}$. The bigger the defect is, the bigger and clearer the hot spot is. With the time passing by, the two defects become more clearly till $0.60 \mathrm{~s}$. Subsequently, the defects become faint because of the lateral thermal diffusion. At last, the surface temperature distribution becomes balanced, and no defect information can be seen by the image (at $30 \mathrm{~s}$ ). It is concluded that the best inspection time is between $0.38 \mathrm{~s}$ and $0.60 \mathrm{~s}$. But three smaller defects could not be observed on the raw images, because of the weak signal produced by the defects, which are also affected by the noise.

(2) Contrast enhancement based on the first-order differential processing of the specimen $2 \#$

The reconstructed image sequence is processed based on the first-order differential, and the 
corresponding results are shown in Fig. 14. It can be seen that the gray value of hot spots at the defect areas experiences the reversal at the moment of $3 \mathrm{~s}$, and the four hot spots, i.e., the four defects, are visible at about $5 \mathrm{~s}$. Therefore, the image before the gray reserval is used to subtract the image after the gray reserval, and the corresponding results are shown in Fig. 15, which indicates that all hot spots of the defects are visible in the image obtained from the reconstructed image.

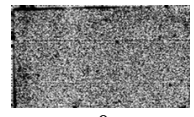

$t=0 \mathrm{~s}$

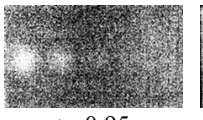

$t=0.95 \mathrm{~s}$

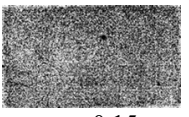

$t=0.15 \mathrm{~s}$

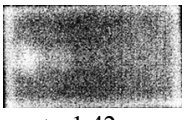

$t=1.42 \mathrm{~s}$

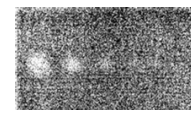

$t=0.38 \mathrm{~s}$

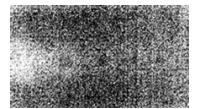

$t=1.88 \mathrm{~s}$

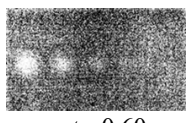

$t=0.60 \mathrm{~s}$

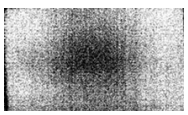

$t=30 \mathrm{~s}$
Fig. 13 Raw thermal images sequence of the specimen $2 \#$.

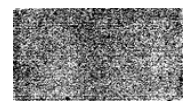

$0.05 \mathrm{~s}$

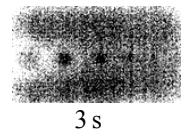

$3 \mathrm{~s}$

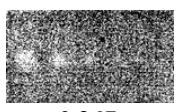

$0.267 \mathrm{~s}$

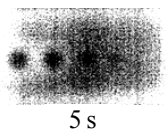

$5 \mathrm{~s}$
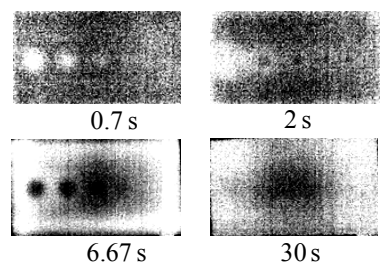

Fig. 14 Thermal images sequence of the specimen $2 \#$ after differential processing.



(a)



(b)
Fig. 15 Results of images processing by subtracting the gray reserval and denoising: (a) result of image before the gray reserval subtracting image after the gray reserval, and (b) denoise.

\section{Conclusions}

In this work, a method based on the combination of the thermal image sequence reconstruction and the first-order differential processing is proposed to enhance the contrast and remove the noise in the thermal images, which is validated by the experimental investigation. The main conclusions are listed as follows:

(1) The piecewise fitting based reconstruction method can not only remove the noise, but also compress the temperature data reaching at $99.1 \%$, which admirably saves the storage space;

(2) The first-order differential processing method can effectively enhance the contrast and improve the thermal image quality.

In summary, the proposed method is proven successfully for the thermal image sequence processing to remove the noise, enhance the contrast, and even compress the data, which can improve the detectability of pulsed infrared thermography greatly.

\section{Acknowledgment}

This work is supported by the National Natural Science Foundation of China (Grant Nos. 51575516 and 51605481) and Xi' an Science and Technology Project (Grant No. 2017089CG/RC052 HJKC001).

Open Access This article is distributed under the terms of the Creative Commons Attribution 4.0 International License (http://creativecommons.org/licenses/by/4.0/), which permits unrestricted use, distribution, and reproduction in any medium, provided you give appropriate credit to the original author(s) and the source, provide a link to the Creative Commons license, and indicate if changes were made.

\section{References}

[1] T. Sakagami, Y. Izumi, and S. Kubo, "Application of infrared thermography to structural integrity evaluation of steel bridges," Journal of Modern Optics, 2010, 57(18): 1738-1746.

[2] V. P. Vavilov, E. Grinzato, P. G. Bison, S. Marinetti, and M. J. Bales, "Surface transient temperature inversion for hidden corrosion characterization: theory and applications," International Journal of Heat and Mass Transfer, 1996, 39(2): 355371.

[3] E. Grinzato, V. Vavilov, P. G. Bison, and S. Marinetti, "Hidden corrosion detection in thick metallic components by transient IR thermography," Infrared Physics \& Technology, 2007, 49: 234-238.

[4] X. P. Maldague, Theory and practice of infrared technology for nondestructive testing. Manhattan, 
USA: Wiley Interscience, 2001: 1-704.

[5] M. Pilla, M. Klein, X. Maldague, and A. Salerno, "New absolute contrast for pulsed thermography," Quantitative Infrared Thermography Journal, 2002: 004: 53-58.

[6] H. D. Benitez, X. Maldague, C. I. Castanedo, H. L. Correa, A. Bendada, and E. F. C. Bravo, "Modified differential absolute contrast using thermal quadrupoles for the nondestructive testing of finite thickness specimens by infrared thermography," in Proceeding of 2006 Canadian Conference on Electrical and Computer Engineering, Ottawa, Ont., Canada, 2007, pp. 1039-1042.

[7] X. W. Guo, W. Shao, G. P. Guo, and Y. Liu, "Image processing algorithms for uneven heating in infrared thermgorahic NDT," Journal of Beijing University of Aeronautics and Astronautics, 2005, 31(11): 1204-1207.

[8] R. Usamentiaga, P. Venegas, J. Guerediaga, L. Vega, and I. Lopez, "Automatic detection of impact damage in carbon fiber composites using active thermography," Infrared Physics \& Technology, 2013, 58(5): 36-46.

[9] Q. J. Tang, C. W. Bu, Y. L. Liu, L. T. Qi, and Z. Y. Yu, "A new signal processing algorithm of pulsed infrared thermography," Infrared Physics \& Technology, 2015, 68: $173-178$.

[10] D. D. Wang, W. Zhang, Z. W. Yang, and G. Tian, "Image enhancement of thermal waving inspection based on independence component analysis," Science Technology and Engineering, 2013, 13(2): 512-515.

[11] T. Liu, W. Zhang, and S. Z. Yan, "A novel image enhancement algorithm based on stationary wavelet transform for infrared thermography to the de-bonding defect in solid rocket motors," Mechanical Systems and Signal Processing, 2015, 62-63: 366-380.

[12] W. Zhang, F. H. Cai, B. M. Ma, and Z. W. Yang, "Quantitative analysis of infrared thermal image defect based on mathematical morphology," Nondestructive Testing, 2009, 31(8): 596-599. 